\title{
Die „soziale Frage als nationale zu rethematisieren"
}

Rezension zu Karina Becker, Klaus Dörre, Peter Reif-Spirek (Hg.) (2018):

Arbeiterbewegung von rechts? Ungleichheit - Verteilungskämpfe - populistische Revolte. Frankfurt/New York: Campus.

Ina Schildbach

Abb. 1 Titel des Buches (Quelle: Verlag Campus)

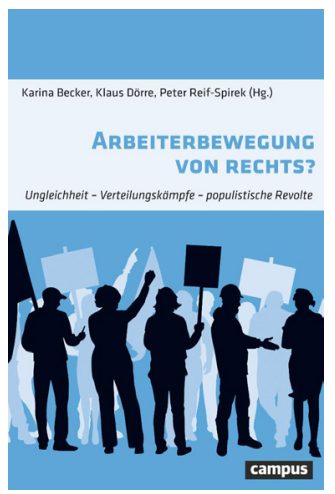

Im Umgang mit Rechtspopulismus und den Wähler_innen rechtspopulistischer Parteien scheuen demokratische Politiker_innen und die Öffentlichkeit nicht vor entpolitisierten, hochmoralischen Urteilen zurück: Die Rede ist von ,Dunkeldeutschland' (ehemaliger Bundespräsident Joachim Gauck) und dem ,Pack' (ehemaliger Vizekanzler Sigmar Gabriel), weshalb wir, die Guten, „dann auch mal vom Sofa hochkommen“ (Justizminister Heiko Maas, Zeit Online, 2.9.2018) müssen. Die Unterscheidung von vermeintlich guten, anständigen Demokrat_innen auf der einen und bösen Populist_innen auf der anderen Seite dient vielleicht dem Bedürfnis der eigenen Erhöhung, erfasst wird das Phänomen jedenfalls nicht. Schließlich agieren Rechtspopulist_innen nicht so, wie sie es tun, ,weil sie böse sind', sondern vielmehr im Bewusstsein, Deutschland besser als ,die Etablierten' regieren zu können. Während also beispielsweise Heiko Maas für sich und alle Demokrat_innen in Anspruch nimmt, das ,Ansehen Deutschlands' gegen Pegida und Co. zu retten, verstehen sich umgekehrt diese selbst als die wahren Patriot_innen.

Auch die Versuche zu erklären, weshalb Menschen rechtspopulistisch wählen, werden häufig ihres politischen Gehalts beraubt: Das Wahlverhalten wird pathologisiert oder psychologisiert, indem beispielsweise von diffus gefühlter Entsicherung gesprochen wird, die einen guten Nährboden für rechtspopulistisches Gedankengut biete. Oder es ist von einem „eigenen Antrieb zur Unterwerfung“(Decker/Brähler 2018: 10) die Rede, die dem „autoritären Charakter" an sich zukomme. Ohne im Einzelnen auf die Aporien der jeweiligen Deutungen einzugehen (vgl. hierzu den Beitrag von Dirk Jörke) sei hier nur darauf verwiesen, dass sich sowohl beim prekären Lebensgefühl als auch dem Willen zur Unterwerfung eine Leerstelle auftut: Inwiefern folgt daraus, dass man sein Kreuz bei Rechtspopulist_innen macht? Weshalb nicht bei der Partei „Die Linke“, die ein Ende der Prekarisierung der Arbeitswelt verspricht? Und warum wird das vermeintliche Unterwerfungsbedürfnis erwartungsgemäß allein durch die AfD, nicht aber der SPD oder einer anderen Partei befriedigt?

Man kann die Aufsatzsammlung Arbeiterbewegung von rechts als den Versuch verstehen, entpolitisierten Erklärungsansätzen, die immer auch 
eine implizite oder explizite Leugnung eines sozioökonomischen Grundes für rechtspopulistisches Wählen darstellen, entgegenzutreten. Das Buch versammelt als Autor_innen nicht nur Wissenschaftler_innen, sondern - und hiervon profitiert diese Aufsatzsammlung aufgrund des behandelten Gegenstandes enorm - Gewerkschaftsfunktionär_innen, die die gesellschaftlichen Entwicklungen und mögliche Antworten aus ihrer Sicht darlegen.

Im ersten Teil („Grundlagen“) argumentiert Klaus Dörre, dass sich die Wahl rechtspopulistischer Parteien weder mit einem psychologischen Reflex noch schlicht mit dem Verweis auf sozioökonomische Lebenslagen erklären lässt. Ausschlaggebend sei, dass es den Rechten gelingt,

„die soziale Frage mit ethno-pluralistisch-nationalistischen Deutungsmustern zu besetzen. Je geringer die Aussichten der von Löhnen abhängigen Klassen sind, in den gesellschaftlichen Verteilungskämpfen eigene Gerechtigkeitsvorstellungen durchzusetzen, desto empfänglicher werden zumindest Teile der Beherrschten für Interpretationen der sozialen Frage, die Gegensätze zwischen oben und unten in Konflikte zwischen innen und außen, zwischen Migranten und einem als kulturell homogen vorgestellten deutschen Volk umdeuten.“ (51)

Dörre geht also davon aus, dass der rechte Erfolg auf einem nationalistischen Urteil über die sozioökonomische Lage beruht: Dass Menschen Angst vor dem Abstieg haben, von Armut bedroht sind oder ohne eigene Betroffenheit die sozial- und arbeitsmarktpolitischen Verhältnisse in Deutschland ungerecht finden, stelle eine Bedingung dar. Zur vollständigen Erklärung sei diese jedoch unzureichend, weil daraus lediglich das Votieren für die Partei mit den meisten sozialen Versprechungen folgen würde. Hinzukommen müsse, dass „die soziale Frage mit ethno-pluralistisch-nationalistischen Deutungsmustern“ (ebd.) besetzt wird. Die soziale Frage als nationale zu reformulieren, bedeutet, dass man sich nicht an der Armut an sich stört, solange das ,eigentliche“ Vorrecht, der Deutschen' gegenüber, den Fremden“ gewahrt bleibt - dies ist der Kern von Dörres ,Umdeutungs-These'.

Dabei unterstellt diese Interpretation, dass die momentane Regierung nicht (mehr) die eigentlichen Interessen, des Volkes' vertritt - nur so lässt sich im rechtspopulistischen Weltbild erklären, dass die eigenen Gerechtigkeitsvorstellungen nichts gelten. In dieser „Krise der politischen Repräsentation“ (104) positionieren sich die rechten Kräfte als die wahre Volksvertretung.

Die soziale Frage muss, will man dem Erfolg der Rechtspopulisten_innen entgegentreten, Dörre zufolge als Klassenfrage reformuliert werden. Stattdessen werde allerorts nur noch von der nebulösen ,Mitte ' gesprochen. Auch in der Wissenschaft werde, folgt man Gudrun Hentges, häufig nicht mehr begrifflich zwischen Schicht und Klasse unterschieden. Das Wissen über Klasse und Klassenbewusstsein sei verloren gegangen, und damit auch das Wissen um die eigene Ausbeutung. „Fehlt es an organisierten gewerkschaftlichen und politischen Zusammenhängen, die solche Kausalmechanismen [Die Reichen sind reich, weil die Armen arm sind, d. h. sie sind reich auf Kosten der Armen, Anm. d. A.] in den Arbeitermilieus verankern könnten, entsteht ein politisches Vakuum, das durch ressentimentgeladene Kausalmechanismen gefüllt werden kann." (65). Dörre zieht hieraus eine eindeutige Konsequenz: „Es kommt darauf an, Rassismus 
nicht nur zu kritisieren, sondern die gesellschaftlichen Verhältnisse zu verändern, die ihn hervorbringen“ (75).

Welche Antworten geben darauf die Gewerkschaften als Akteure, die höhere Löhne und bessere Arbeitsbedingungen erkämpfen müssten? Im zweiten Teil des Bandes („Empirische Befunde: Arbeiter_innen, Autoritarismus, Gewerkschaften") entwickeln unter anderem Annelie Buntenbach, Mitglied des DGB-Bundesvorstandes, und Hans-Jürgen Urban, geschäftsführendes Vorstandsmitglied der IG Metall, ihre Thesen zur „Arbeiterbewegung von rechts“. Während die in dem Band dargelegten empirischen Ergebnisse die entwickelte Analyse stützen, [1] bestreitet Buntenbach die Charakterisierung ,Arbeiterbewegung“. „Die Entzündungsherde für diese Entwicklung liegen ja nicht im Betrieb oder in den klassischen Anliegen der Arbeiterbewegung: Durchsetzung besserer Löhne und Arbeitsbedingungen, Begrenzung der Arbeitszeit“ (170), was sie mit dem vermeintlich neoliberalen Charakter der AfD begründet. Es lässt sich diskutieren, ob sich in der AfD - zweifellos als marktradikale Partei gegründet - der neoliberale oder der sozialstaatlich orientierte Flügel durchsetzt. Jedoch kann kaum bestritten werden, dass es letzteren gibt, und die Partei im Zusammenhang mit der Migrations- und Flüchtlingspolitik stark sozialprotektionistisch agitiert (vgl. hierzu auch Schildbach 2019). „Sozialpolitik und Zuwanderung“ lautet dementsprechend ein Punkt im Wahlprogramm der Partei (AfD 2017).

Buntenbach bezweifelt, dass der Erfolg der AfD in dieser Argumentationsweise begründet liegt: „Wählen sie [die AfD-affinen Arbeitnehmer_innen, Anm. d. A.] als Arbeitnehmer eine extrem neoliberale Partei wie die AfD oder tun sie das als Trägerinnen rassistischer, homophober oder chauvinistischer Haltungen?“ (170). Damit trennt sie, was den Analysen Dörres, Martin Kronauers und Hentges zufolge nicht zu separieren sei: Als abhängig Beschäftigte denken Menschen nationalistisch und wählen eine Partei, die dem vermeintlichen Vorrecht des deutschen Arbeitnehmers dient. Konsequent auch, dass Buntenbach auf Basis ihrer Diagnose die Gewerkschaften nicht primär in ihrer klassischen Rolle, sondern in bildungs- und zivilgesellschaftlicher Hinsicht gefordert sieht (173ff.), während Richard Detje und Dieter Sauer dafür plädieren, dem Rechtspopulismus auch in den Betrieben den Boden zu entziehen.

Auch der Beitrag Hans-Jürgen Urbans steht konträr zu Buntenbachs Einschätzung: Er argumentiert dafür, ,das soziale Ereignis- und Erlebnisfeld der kapitalistischen Erwerbsarbeit in den Blick zu nehmen und nach arbeitsweltlichen Ursachen rechtspopulistischer Einstellungen zu fragen" (183) und kritisiert die „Arbeitsweltvergessenheit der Populismusforschung“ (189). Dementsprechend ist es dem Autor zufolge auch möglich, dass gewerkschaftliche Macht durch eine interessenpolitische Konkurrenz von rechts - deren Existenz Buntenbach vermutlich bestreiten würde - weiter erodiert.

Im dritten Teil („Internationale Perspektiven: Arbeiter_innen und Rechtspopulismus in Europa“) wird am Beispiel der FPÖ (Freiheitliche Partei Österreichs) sowie der polnischen PiS (Recht und Gerechtigkeit) untersucht, inwiefern rechtspopulistische Parteien tatsächlich eine Sozialund Arbeitsmarktpolitik im Sinne der abhängig Beschäftigten verfolgen. Dabei werden vor allem in Bezug auf Österreich Widersprüche zwischen Programmatik und Regierungspolitik der „sozialen Heimatpartei“, wie sich 
die FPÖ selbst nennt, deutlich. Dass sie die Sozialpolitik jedoch völkischen Kriterien unterordnet (250), stellt sicherlich keine Aporie der Partei dar, ist der Gegenstand ihrer Sorge doch selbstredend nicht die Armut an sich, sondern die der autochthonen Bürger_innen.

Der vierte Teil („Kontroversen: Klassen- und/oder Identitätspolitik?“) greift Debatten innerhalb der Linken auf. Hervorzuheben ist hier vor allem der Beitrag von Jörke, der sich mit den eingangs erwähnten alternativen Erklärungsansätzen beschäftigt. Auch er beklagt eine Moralisierung der wissenschaftlichen Auseinandersetzung mit Rechtspopulist_innen, die den Eindruck erweckt, ,dass es weniger um die Überzeugungskraft des Arguments als um die Frage ging, ob man auf der richtigen Seite steht“" (288). Dabei, so argumentiert der Autor, verkennen moralisierende und sozialpsychologische Ansätze, dass Menschen mit rechtspopulistischer Einstellung „,öllig rational“ (294) handeln: Sie sehen sich von der Globalisierung bedroht und wählen diejenigen Parteien, die ihnen Schutz vor Zugewanderten und eine Verteidigung des Sozialstaats versprechen. Jörke zufolge zeige etwa die PiSRegierung in Polen, dass die unteren Klassen nicht gegen ihre Interessen stimmen - und dies gelte umso mehr, als linke Parteien an einer liberalen Migrationspolitik festhalten.

Unklar bleibt jedoch, was Jörke - abgesehen von der Ablehnung einer Moralisierung der Debatte - daraus folgert: Soll die Linke ihre „kosmopolitischen Werte" (294) aufgeben und die Einwanderungspolitik der Rechten übernehmen? Gerade wenn man die Erkenntnis ernst nimmt, dass es - immanent gedacht! - rational sein kann, eine Partei wie die PiS zu wählen, kann die Antwort doch kaum in Verständnis und argumentativer Kapitulation liegen. Stattdessen gilt es, das nationale Denken anzugreifen und die gemeinsamen Interessen der globalen Arbeiterklasse herauszuarbeiten - insofern ,rückzuentwickeln', was Didier Eribon in einem Interview mit der Süddeutschen Zeitung (Rühle 2016) beklagt: „Ich habe in meiner Familie gesehen, wie sich das Weltbild verschoben hat. Aus: ,Wir Arbeiter gegen die Bourgeoisie“ wurde allmählich, Wir Franzosen gegen die Migranten'. Heute ist das völlig verfestigt.“

Gerade angesichts sich widersprechender Analysen, die durchaus fruchtbar unterschiedliche Positionen - vor allem auch von Gewerkschaftsseite zum Thema darlegen, hätte man sich ein abschließendes Fazit gewünscht, das sich vonseiten der Herausgeber argumentativ den kontroversen Ansichten stellt. Dennoch kann die Lektüre uneingeschränkt empfohlen werden - angesichts der gesellschaftlichen Relevanz des Themas nicht nur den hierzu Forschenden, den Multiplikatoren_innen in der politischen Bildungsarbeit und Gewerkschaftern_innen, sondern allen politisch Denkenden.

\section{Endnoten}

[1] So bejahen einer repräsentativen Umfrage zufolge 87,9 Prozent der AfD-Sympathisanten und 62 Prozent der Anhänger der restlichen Parteien die Aussage, dass ihnen die steigende Armut in Deutschland Angst mache (siehe den Beitrag von Lorenzen et al.). „Dass ich selbst arm werde“, befürchten 69,7 Prozent der AfD-Anhänger und 38,4 Prozent der anderen Parteien (ebd.; siehe auch den Beitrag von Bose/Köster/Lütten). 


\section{Autor_innen}

Ina Schildbach ist Politikwissenschaftlerin. Ihre Arbeitsschwerpunkte sind Armuts- und Sozialstaatsforschung sowie Migrationspolitik.

ina.schildbach@th-nuernberg.de

\section{Literatur}

AfD (2017): Programm für Deutschland. Wahlprogramm der Alternative für Deutschland für die Wahl zum Deutschen Bundestag am 24. September 2017. https://www.afd.de/ wahlprogramm/ (letzter Zugriff am 17.12.2018).

Becker, Karina / Dörre, Klaus / Reif-Spirek, Peter (Hg.) (2018): Arbeiterbewegung von rechts? Ungleichheit - Verteilungskämpfe - populistische Revolte. Frankfurt/New York: Campus.

Buntenbach, Annelie (2018): Keine Alternative zur Arbeiterbewegung: Die Anziehungskraft der AfD für Arbeitnehmer und Arbeitnehmerinnen -- Eine Herausforderung für Gewerkschaften. In: Becker, Karina / Dörre, Klaus / Reif-Spirek, Peter (Hg.), Arbeiterbewegung von rechts? Ungleichheit - Verteilungskämpfe - populistische Revolte. Frankfurt/New York: Campus, 169-182.

Decker, Oliver / Brähler, Elmar (Hg.) (2018): Flucht ins Autoritäre. Rechtsextreme Dynamiken in der Mitte der Gesellschaft. Gießen: Psychosozial-Verlag.

Dörre, Klaus (2018): In der Warteschlange. Rassismus, völkischer Populismus und die Arbeiterfrage. In: Becker, Karina / Dörre, Klaus / Reif-Spirek, Peter (Hg.), Arbeiterbewegung von rechts? Ungleichheit - Verteilungskämpfe - populistische Revolte. Frankfurt/New York: Campus, S. 49-80.

Flecker, Jörg / Altreiter, Carina / Schindler, Saskia (2018): Erfolg des Rechtspopulismus durch exkludierende Solidarität? Das Beispiel Österreich. In: Becker, Karina / Dörre, Klaus / Reif-Spirek, Peter (Hg.), Arbeiterbewegung von rechts? Ungleichheit - Verteilungskämpfe - populistische Revolte. Frankfurt/New York: Campus, 245-256.

Hentges, Gudrun (2018): Die populistische Lücke: Flucht, Migration und Neue Rechte. In: Becker, Karina / Dörre, Klaus / Reif-Spirek, Peter (Hg.), Arbeiterbewegung von rechts? Ungleichheit - Verteilungskämpfe - populistische Revolte. Frankfurt/New York: Campus, 101-116.

Jörke, Dirk (2018): Moralisierung und die Instrumentalisierung sozialpsychologischer Erklärungen. In: Becker, Karina / Dörre, Klaus / Reif-Spirek, Peter (Hg.), Arbeiterbewegung von rechts? Ungleichheit - Verteilungskämpfe - populistische Revolte. Frankfurt/ New York: Campus, 287-296.

Kronauer, Martin (2018): Warum und wie die Linke heute für soziale Gerechtigkeit streiten muss. In: Becker, Karina / Dörre, Klaus / Reif-Spirek, Peter (Hg.), Arbeiterbewegung von rechts? Ungleichheit - Verteilungskämpfe - populistische Revolte. Frankfurt/New York: Campus, 81-100.

Rühle, Alex (2016): Wir müssen uns viel lauter einmischen. Interview mit dem Soziologen Didier Eribon, in: Süddeutsche Zeitung, 24.11.2016.

Schildbach, Ina (2019): „Die neue deutsche Soziale Frage“ - Armut und Sozialstaatsversagen als Grund für rechtspopulistischen Erfolg? In: Lukas Boehnke / Malte Thran / Jacob Wunderwald (Hg.), Rechtspopulismus im Fokus. Theoretische und praktische Herausforderungen für die politische Bildung. Berlin: Springer VS, 73-88.

Urban, Hans-Jürgen (2018): Rechtspopulismus, Gewerkschaften und Demokratiepolitik: Soziologische Befunde und transformatorische Optionen. In: Becker, Karina / Dörre, Klaus / Reif-Spirek, Peter (Hg.), Arbeiterbewegung von rechts? Ungleichheit - Verteilungskämpfe - populistische Revolte. Frankfurt/New York: Campus, 183-196. 